\title{
Fragility and correlated dynamics in supercooled liquids
}

Cite as: J. Chem. Phys. 153, 124501 (2020); https://doi.org/10.1063/5.0015091

Submitted: 26 May 2020 • Accepted: 30 August 2020 • Published Online: 22 September 2020

(D) Atreyee Banerjee and (D) David J. Wales

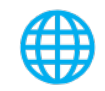

\section{ARTICLES YOU MAY BE INTERESTED IN}

Non-adiabatic quantum dynamics without potential energy surfaces based on secondquantized electrons: Application within the framework of the MCTDH method The Journal of Chemical Physics 153, 154110 (2020); https://doi.org/10.1063/5.0028116 Liquid-liquid transition and polyamorphism

The Journal of Chemical Physics 153, 130901 (2020); https://doi.org/10.1063/5.0021045

Universal correlations between the fragility and interparticle repulsion of glass-forming liquids

The Journal of Chemical Physics 153, 124507 (2020); https://doi.org/10.1063/5.0014457

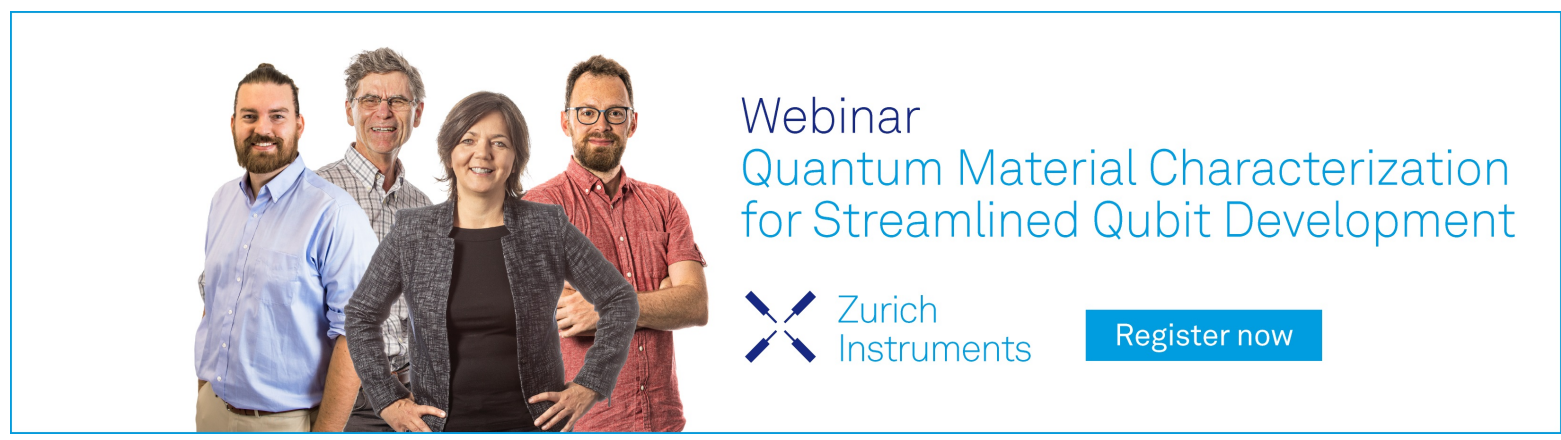




\title{
Fragility and correlated dynamics in supercooled liquids
}

\author{
Cite as: J. Chem. Phys. 153, 124501 (2020); doi: 10.1063/5.0015091 \\ Submitted: 26 May 2020 - Accepted: 30 August 2020 • \\ Published Online: 22 September 2020
}

Atreyee Banerjee $^{1,2, a)}$ (D) and David J. Wales ${ }^{1, b)}$ (D)

\begin{abstract}
AFFILIATIONS
${ }^{1}$ Department of Chemistry, University of Cambridge, Lensfield Road, Cambridge CB2 1EW, United Kingdom

${ }^{2}$ Max Planck Institute for Polymer Research, 55128 Mainz, Germany
\end{abstract}

\author{
author to whom correspondence should be addressed: batreyee89@gmail.com \\ b)dw34@cam.ac.uk
}

\begin{abstract}
A connection between the super-Arrhenius behavior of dynamical properties and the correlated dynamics for supercooled liquids is examined for a well known glass forming binary Lennard-Jones mixture and its repulsive counterpart, the Weeks-Chandler-Andersen potential, over a range of densities. When considering short time nonergodic trajectory segments of a longer ergodic trajectory, we observe that, independent of the potentials and densities, the apparent diffusivity follows Arrhenius behavior until low temperatures. Comparing the two potentials, where the ergodic diffusivities are known to be rather different, we find that the short-time nonergodic part is similar throughout the temperature range. By including a correlation factor in the nonergodic diffusivity, a rescaled value is calculated, which provides a reasonable estimate of the true ergodic diffusivity. The true diffusion coefficient and the correction factor collapse to a master plot for all densities at any given time interval. Hence, our results confirm a strong connection between fragility and dynamical correlation.
\end{abstract}

Published under license by AIP Publishing. https://doi.org/10.1063/5.0015091

\section{INTRODUCTION}

Liquids can enter a metastable supercooled state on fast cooling. The viscosity and relaxation time increase rapidly, but the structure of the supercooled state appears to be unaltered with respect to the high temperature liquid state. ${ }^{1}$ Thus, the origin of the sluggish dynamics still remains an open question. At higher temperatures, the dynamical observable properties $(X)$, such as viscosity, diffusion coefficient, and relaxation time, follow Arrhenius behavior, where $X \propto \exp ( \pm E / T)$ for a constant $E$, which is interpreted as an activation energy. However, below the onset temperature of glassy dynamics, the dynamical properties may follow super-Arrhenius behavior. The Vogel-Fulcher-Tammann (VFT) equation commonly fits the behavior quite well at low temperatures, ${ }^{2-4}$ where $X \propto \exp ( \pm E /$ $\left(T-T_{g}\right)$ ), with $T_{g}$ being the glass transition temperature where the system is eventually arrested in a non-crystalline jammed state. Alternative fits without singularities can also be used.

Based on the rate of slowdown of the dynamical properties, supercooled liquids are categorized into two classes as "strong" and "fragile." $", 9$ These properties follow Arrhenius behavior until a much lower temperature for the "strong" liquids, whereas they deviate faster for "fragile" liquids. Network-forming glass formers, such as silica and germanium oxides, are usually strong materials, while systems such as orthoterphenyl and Lennard-Jones (LJ) binary mixtures at high densities are often classed as fragile glass formers. $^{10-13}$

As for any system, the dynamics of supercooled liquids become increasingly heterogeneous on a finite observation time scale at lower temperatures, both experimentally and in computer simulations. ${ }^{14-17}$ The structural relaxation time follows a non-exponential two-step decay on cooling the liquid. ${ }^{1}$ There have been extensive studies to connect the longer $\alpha$-relaxation time scale to the dynamical heterogeneity of the system. ${ }^{18-20}$ The positive value of nonGaussian parameter $\alpha_{2}=\frac{3\left\langle\Delta r^{4}(t)\right\rangle}{5\left\langle\Delta r^{2}(t)\right\rangle^{2}}-1$ at low temperatures reflects the dynamical heterogeneity, which goes to zero at long time scales for an ergodic trajectory. ${ }^{21}$ The ergodic self-diffusion coefficient is usually obtained from the Einstein relationship in the $t \rightarrow \infty$ limit for the supercooled state, and it may exhibit super-Arrhenius behavior at low temperatures. The temperature dependence of dynamic 
observables, such as the structural relaxation time and the diffusion coefficient, is often interpreted via the Adam-Gibbs relationship, ${ }^{22}$ which involves a configurational entropy of some sort. Rabani et al. estimated a residence time scale for cage rattling and connected that to the non-exponential decay. ${ }^{23}$ The cage-breaking time scale has been connected to the super-Arrhenius behavior. ${ }^{2}$ Doliwa and Heuer defined the superstructure of the landscape in terms of metabasins, which were associated with longer time scale dynamics. ${ }^{25,26}$ Although many theories of the glass transition consider long time structural relaxation, Ngai et al. have shown that this observable originates from its precursor short time $\beta$-relaxation. ${ }^{27}$ More recently, the dynamical heterogeneity has also been connected to the shorter time $\beta$-relaxation of the system. ${ }^{28,29}$ The importance of the short time dynamics to the growing length scales and the heterogeneity has been established in experiments and simulations. ${ }^{30}$ The present approach provides a different perspective from treatments based on the energy landscape, where the dynamics are coarsegrained in terms of transitions between local minima. The correlation effect we analyze here in terms of explicit dynamics is consistent with the need to consider productive cage-breaking rearrangements in analyzing the overall diffusion. ${ }^{12,13,24,31}$ Atoms must change their local environment in terms of the surrounding nearest-neighbors to achieve diffusion, but some case-breaking processes are rapidly reversed. Hence, negative correlation also manifests itself when diffusion is broken down into transitions between local minima on the potential energy landscape.

In previous work, ${ }^{5,32}$ we calculated a time-dependent diffusion constant for a nonergodic subset of an ergodic trajectory and found that even for a fragile system, it followed Arrhenius behavior. We observed that this short time diffusion constant can be connected to the true super-Arrhenius behavior by accounting for a negative correction factor, which depends on the average angle between the steps in successive time intervals. The value of the negative correction increases on lowering the temperature, thus establishing a connection between the negative correlation and the dynamics. The correlation is also found to be important for a strong glass forming liquid, such as $\mathrm{SiO}_{2}{ }^{13}$ The number of available diffusive pathways appears to decrease at lower temperatures. ${ }^{24}$

There have been extensive studies comparing systems based on the Lennard-Jones (LJ) potential and its repulsive counterpart, the Weeks-Chandler-Andersen (WCA) form. ${ }^{33-36}$ The dynamical properties of the two systems are distinct, but they appear to have similar structures. ${ }^{33}$ Furthermore, it has been established that the difference in dynamical properties can be connected to the entropy. ${ }^{36-39}$ Recently, based on a machine learned softness field, a structure-dynamics correlation has been quantified. ${ }^{40}$ It was shown that the LJ system is more fragile than WCA at low densities. ${ }^{34,41}$ However, as the density increases, both the dynamical and thermodynamic properties are comparable for LJ and WCA systems, and the fragilities are similar at higher densities. ${ }^{41}$ LJ systems obey a density-temperature scaling over a wide range of densities, which breaks down for WCA at low densities. ${ }^{35,42}$

In the present contribution, we calculate the short time nonergodic diffusion coefficients ${ }^{5,32}$ for LJ and WCA systems at various densities. We observe that for both of them, the nonergodic diffusion coefficient overestimates the actual diffusion constant, without the negative correction factor. However, on including this factor, super-Arrhenius behavior is recovered. A comparison between the dynamical properties of LJ and WCA systems at lower densities indicates that, even though the two systems behave differently at long time scales, they behave similarly at short time scales. However, at higher densities, they behave similarly throughout the observable time range. The long time diffusion coefficient and the negative correlation are found to follow a master curve for both systems at all the densities considered and all the time windows.

This paper is organized as follows: The simulation details are given in Sec. II. In Sec. III, we present our results. Section IV contains a brief conclusion.

\section{SIMULATION DETAILS}

We performed molecular dynamics (MD) simulations of a 256-particle binary mixture of $204 \mathrm{~A}$ and $52 \mathrm{~B}$ atoms, interacting according to the LJ potential and its repulsive counterpart (WCA) in a periodically repeated cell. ${ }^{43,44}$ The interatomic pair potential between species $\alpha$ and $\beta$, with $\alpha, \beta=\mathrm{A}, B, U_{\alpha \beta}(r)$ is described by a shifted and truncated LJ potential,

$$
U_{\alpha \beta}(r)= \begin{cases}U_{\alpha \beta}^{(\mathrm{LJ})}\left(r ; \sigma_{\alpha \beta}, \epsilon_{\alpha \beta}\right)-U_{\alpha \beta}^{(\mathrm{LJ})}\left(r_{\alpha \beta}^{(c)} ; \sigma_{\alpha \beta}, \epsilon_{\alpha \beta}\right), & r \leq r_{\alpha \beta}^{(c)} \\ 0, & r>r_{\alpha \beta}^{(c)},\end{cases}
$$

where $U_{\alpha \beta}^{(\mathrm{LJ})}\left(r ; \sigma_{\alpha \beta}, \epsilon_{\alpha \beta}\right)=4 \epsilon_{\alpha \beta}\left[\left(\sigma_{\alpha \beta} / r\right)^{12}-\left(\sigma_{\alpha \beta} / r\right)^{6}\right]$ and $r_{\alpha \beta}^{(c)}$ is equal to the position of the minimum of $U_{\alpha \beta}^{(\mathrm{LJ})}$ for the WCA systems. Length, temperature, and time are given in units of $\sigma_{\mathrm{AA}}, k_{B} T / \epsilon_{\mathrm{AA}}$, and $\tau=\sqrt{ }\left(m_{\mathrm{A}} \sigma_{\mathrm{AA}}^{2} / \epsilon_{\mathrm{AA}}\right)$, respectively. Here, we have simulated the Kob-Andersen model ${ }^{43}$ with interaction parameters $\sigma_{\mathrm{AA}}=1.0$, $\sigma_{\mathrm{AB}}=0.8, \sigma_{\mathrm{BB}}=0.88, \epsilon_{\mathrm{AA}}=1, \epsilon_{\mathrm{AB}}=1.5, \epsilon_{\mathrm{BB}}=0.5$, and $m_{\mathrm{A}}=m_{\mathrm{B}}=1.0$. We performed $\mathrm{MD}$ simulations in the canonical ensemble (NVT) for a Nosé-Hoover thermostat with integration time step $0.005 \tau$ using LAMMPS. ${ }^{45}$ The time constants for the Nosé-Hoover thermostat are taken to be 100 time steps. We have studied the systems at densities $\rho$ from 1.2, 1.4, and 1.6 for both LJ and WCA potentials.

\section{RESULTS}

At high temperatures, supercooled liquids generally follow Arrhenius behavior, which can change to super-Arrhenius behavior on cooling. To understand the origin of this transition for different glass forming liquids, we follow the analysis in previous work ${ }^{5,32}$ and define a non-ergodic diffusion constant $D(\tau, T)$. A similar time window analysis for the Kolmogorov entropy to quantify information loss in the chaotic dynamics has been reported earlier. ${ }^{46,47}$ The actual diffusion coefficient, $D(T)$, is calculated using the Einstein relationship in the ergodic limit. The nonergodic diffusion constant $D(\tau, T)$ is calculated over shorter, nonergodic time intervals, $\tau$, and we consider the average value over an ergodic trajectory. The true diffusion coefficient $D(T)$ is recovered when the interval $\tau$ is large enough. $D(T)$ is calculated as

$$
D(T)=\lim _{t \rightarrow \infty} \frac{1}{6 t}\left\langle\Delta \mathbf{r}_{i}(t)^{2}\right\rangle
$$


where $\langle\cdot\rangle$ denotes the ensemble average over atom $i$. If the total trajectory is divided into $m$ shorter time intervals, $\tau$, then we define the diffusion constant for time scale $\tau$ as,

$$
D(\tau, T) \equiv \lim _{t \rightarrow \infty} \frac{1}{6 t}\left\langle\Delta \mathbf{r}_{i}(t, \tau)^{2}\right\rangle
$$

where the mean squared displacement $\Delta \mathbf{r}_{i}(t, \tau)^{2}=\sum_{j=1}^{m} \Delta \mathbf{r}_{i}(j)^{2}$ with $m \tau=t$, and the displacement of particle $i$ in time interval $j$ is $\Delta \mathbf{r}_{i}(j)=\mathbf{r}_{i}(j \tau)-\mathbf{r}_{i}(j \tau-\tau)$.

For smaller values of $\tau$, the diffusion coefficient is generally overestimated because the correlation between different time intervals is neglected in the above expression. The true squared displacement can be written in terms of the displacements in successive time windows $\Delta \mathbf{r}_{i}(j)$ as

$$
\begin{aligned}
\Delta \mathbf{r}_{i}(t)^{2} & =\sum_{j=1}^{m} \Delta \mathbf{r}_{i}(j)^{2}+2 \sum_{j<k} \Delta \mathbf{r}_{i}(j) \cdot \Delta \mathbf{r}_{i}(k) \\
& =\sum_{j=1}^{m} \Delta \mathbf{r}_{i}(j)^{2}+2 \sum_{j<k} \Delta r_{i}(j) \Delta r_{i}(k) \cos \theta_{j, k}(i) .
\end{aligned}
$$

Here, $\theta_{j k}(i)$ is the angle between the displacement vectors for atom $i$ in intervals $j$ and $k$, and $\Delta r_{i}(j)$ represents the magnitude of $\Delta \mathbf{r}_{i}(j)$. The second term in Eq. (4) is neglected to calculate $D(\tau, T)$ in Eq. (3), but we will see that the correlation term $\cos \theta_{j, k}(i)$ is essential to calculate the true diffusion constant if $\tau$ is small.

In Fig. 1, we plot $D(\tau, T)$ for the LJ and WCA systems at $\rho=1.2$. At this density, LJ is more fragile than WCA. ${ }^{18,36}$ As $\tau$ increases, we recover the true long time diffusion coefficients for both systems. The figure shows that the super-Arrhenius behavior reappears at larger values of $\tau$. At high temperatures, for both systems, ergodicity is achieved even with small $\tau$ values, suggesting that the strength of the correlation decreases at higher temperatures. However, at low temperatures and small $\tau$, the approximation significantly overestimates the diffusion constant because correlation is neglected. We have calculated $D(\tau, T)$ for different densities and find similar behavior (see the supplementary material).

To understand the effect of the negative correction as a function of temperature, we examine the correlation factor, which depends on the average angle between steps [second term of Eq. (4)]. Since the correction is important at low temperatures, $D(\tau, T)$ is an overestimate at lower temperatures for both systems (Fig. 1). To investigate this effect, we plot the average of $\cos \theta_{j, k}$ over atoms and over the trajectory (Fig. 2) as a function of $n$, where $k=j+n$, for a particular time window $\tau=100$. We find that the mean $\cos \theta_{j, k} \approx 0$ for $n>1$, which indicates that the directions are uncorrelated at larger intervals. However, for the adjacent interval, the displacements are negatively correlated up to a time threshold, and the magnitude of the negative correction increases on lowering the temperature. The existence of negative correlation was demonstrated in earlier studies, ${ }^{5,25,32,48}$ in agreement with the present work. This behavior is independent of the nature of the potential or the density. A vanishing correction term is consistent with a random walk, where the angle and magnitude of the displacements are independent and separable. The magnitudes of the negative corrections for different densities are given in the supplementary material. (a) LJ, $\rho=1.2$

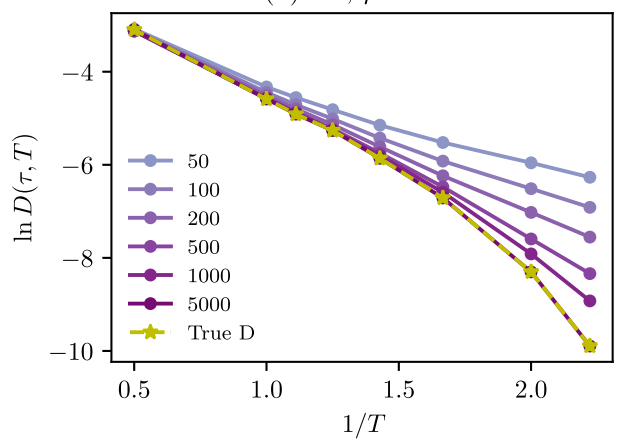

(a) LJ, $\rho=1.2$

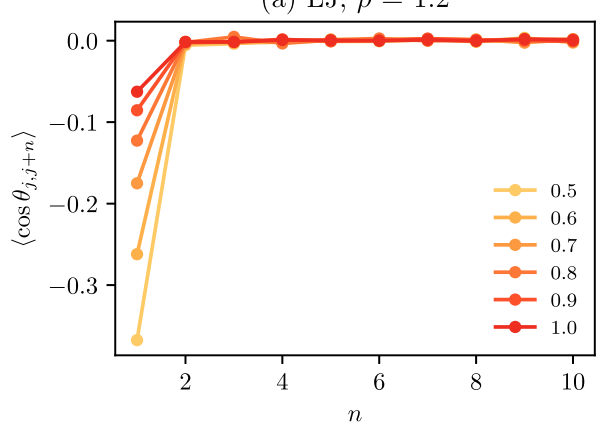

(b) WCA, $\rho=1.2$

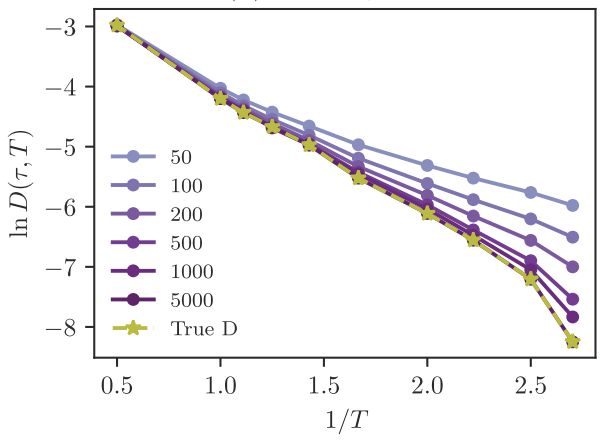

(b) WCA, $\rho=1.2$

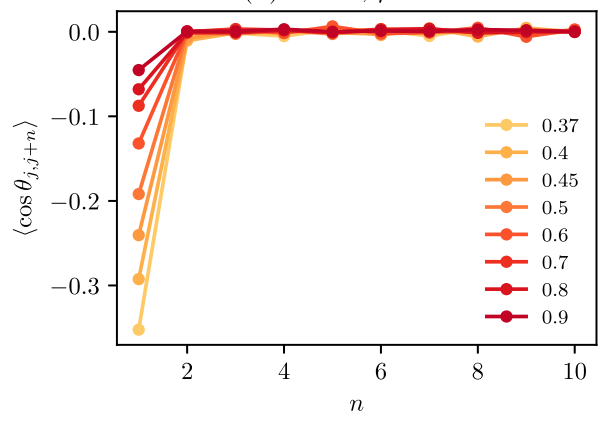

FIG. 1. The time-dependent diffusion coefficients $D(\tau, T)$ for $\mathrm{LJ}$ and WCA systems at $\rho=1.2$. We have calculated $D(\tau$, $T$ ) for different time windows $\tau=50,100$, $200,500,1000$, and $5000 \mathrm{MD}$ steps with each time step $d t=0.005$ in reduced units. We observe at $\tau=5000$ that $D(\tau$, $T$ ) is practically converged to the true diffusion constant calculated using Eq. (2). For both systems, at high temperatures, $D(\tau, T)$ is a reasonable estimate, but at lower temperatures with shorter time windows, it is significantly too large.

FIG. 2. $\cos \theta_{j, j+n}$ averaged over atoms and over the trajectory for different temperatures at $\tau=100$. The plot shows that the direction of motion for non-adjacent time intervals is essentially uncorrelated above a certain time threshold. For both the LJ and WCA systems, the average decreases on lowering the temperature. 
(a) LJ, $\rho=1.2, T=0.70$

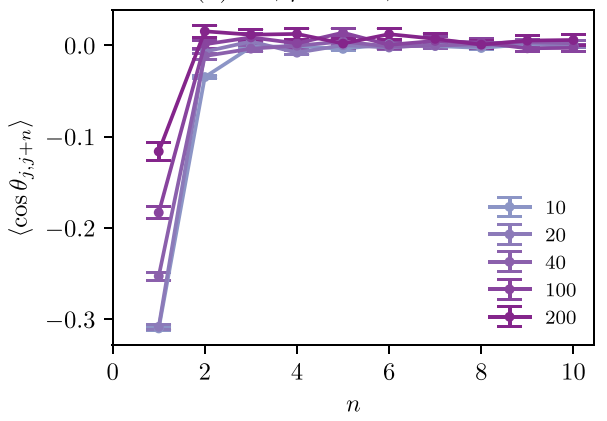

(b) WCA, $\rho=1.2, T=0.70$

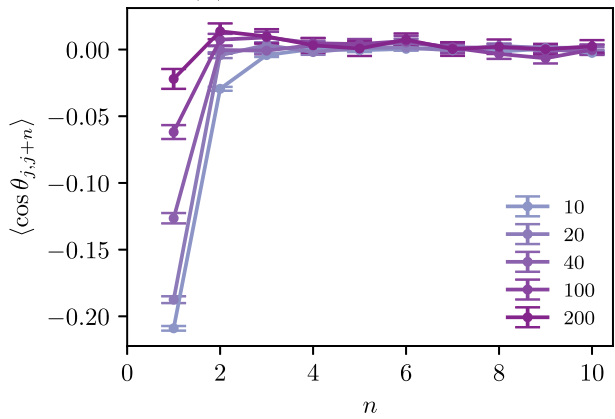

FIG. 3. $\cos \theta_{j, j+n}$ averaged over atoms and over the trajectory increases with decreasing $\tau$. This result suggests that the negative correlation is mostly important for shorter time windows. For longer windows, $D(\tau, T)$ is already in good agreement with the actual relaxation time of the system. The error bars associated with the mean of the average $\left\langle\cos \theta_{j, j+n}\right\rangle$ are shown in the figure.
To measure the time scale for which the negative correction survives, we compare the correlation term for different time intervals. We plot $\left\langle\cos \theta_{j, j+1}\right\rangle$, averaged over atoms and the trajectory, for both systems at a fixed temperature, $T=0.70$, as shown in Fig. 3 . The magnitude of $\left\langle\cos \theta_{j, j+1}\right\rangle$ increases with decreasing $\tau$ and decreasing temperature. We set the error bar of our calculation as $2 \sigma$, where $\sigma$ is the standard deviation. The small positive values for $\left\langle\cos \theta_{j, j+1}\right\rangle$ with $n>1$ are simply statistical noise. We observe that the anticorrelation factor between short time windows is significant, so incorporation of this term is necessary to estimate $D(T)$. However, for larger time windows, the magnitude of the anticorrelation term decreases, and consequently, $D(\tau, T)$ is a better estimate of true diffusion constant. We also observe that the values are higher for the LJ system compared to WCA when compared at a given temperature, which indicates that the negative correction is more important for LJ. This result is consistent with earlier observations that diffusion in the LJ system is slower compared to its repulsive counterpart at $\rho=1.2$. $^{3}$ In Fig. 3, we find that the correction function for $n=1$ is negative up to a certain time threshold and eventually decays to zero when calculated for long time windows. To quantify this effect, we have plotted $\left\langle\cos \theta_{j, j+1}\right\rangle$ as a function of the number of MD steps in Fig. 4. It is clear that the correction goes to zero within the error bar as time increases. Here, the error bars are defined in the same way as for Fig. 3. For low temperatures, the correction survives to longer times, while at higher temperatures, the function decorrelates in shorter time windows. The time scale associated with the decorrelation, where $\left\langle\cos \theta_{j, j+1}\right\rangle \approx 0$, increases at lower temperatures and is closely connected to the $\alpha$-relaxation time/caging of the system. In the inset of Figs. 4(a) and 4(b), we plot the mean square displacement as a function of temperature, which decreases at lower temperatures. The time scale associated with decorrelation of $\left\langle\cos \theta_{j, j+1}\right\rangle$ and the magnitude of the function $\left\langle\cos \theta_{j, j+1}\right\rangle$ in any chosen time window both increase on lowering the temperature. These effects are essentially connected to the growing time scale and length scale, respectively, on vitrification.

To estimate an approximate mean field-like diffusion, we assume that $m$ in Eq. (4) is large, and the magnitude of displacements in the adjacent steps is similar, so that only the average behavior of $\cos \theta_{j k}$ is important, giving

$$
\left\langle\Delta \mathbf{r}^{2}(t)\right\rangle \approx \sum_{j=1}^{m}\left\langle\Delta \mathbf{r}_{i}(j)^{2}\right\rangle\left(1+2\left\langle\cos \theta_{j, j+1}\right\rangle\right) .
$$

This approximation is valid in a mean field limit, where we assume that the displacement for every atom in every interval has the same magnitude. This simplification allows us to derive a new expression for the diffusion coefficient, $D^{*}(\tau, T)$, where super-Arrhenius (a) LJ, $\rho=1.2$

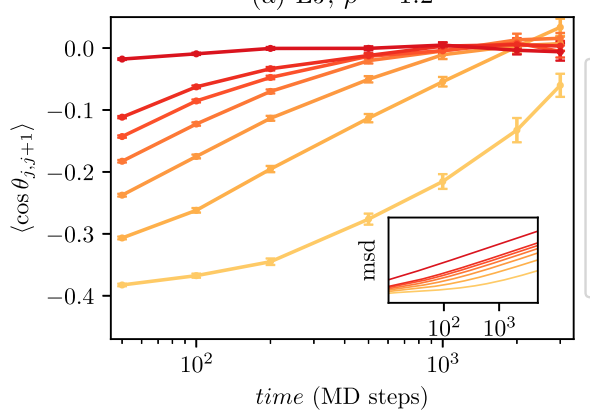

(b) WCA, $\rho=1.2$

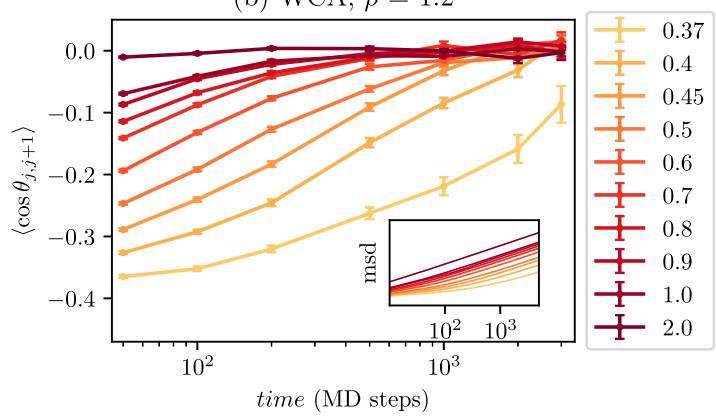

FIG. 4. $\left\langle\cos \theta_{j, j+1}\right\rangle$ and the mean squared displacement (msd) at $\rho=1.2$ for different values of the time window $(\tau)$ and for a range of temperatures. At high temperatures, the function goes to zero for fewer time steps, whereas the lower temperature requires more time to decorrelate. Comparing the mean squared displacement (msd) in the inset figures suggests that the time scale for decorrelation of $\left\langle\cos \theta_{j, j+1}\right\rangle$ is connected to the caging time scale. 


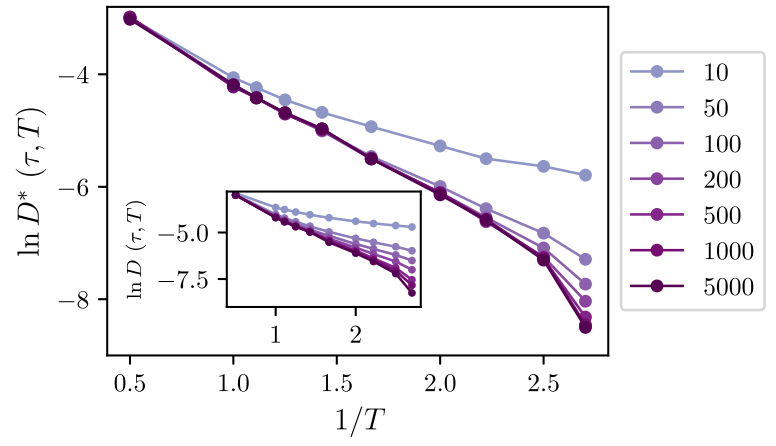

FIG. 5. The mean-field type approximation $D^{*}(\tau, T)$ for the WCA system at $\rho=1.2$ for different values of the time window $(\tau)$. Inset: $D(\tau, T)$ for the same temperature range.

behavior is recovered for different time intervals using the mean correction term ${ }^{5,32}$

$$
D^{*}(\tau, T)=D(\tau, T)\left(1+2\left\langle\cos \theta_{j, j+1}\right\rangle\right) .
$$

In Fig. 5, we plot the nonergodic diffusion coefficients $D^{*}(\tau, T)$ and $D(\tau, T)$ (inset) for WCA systems at a density of 1.2. The plot shows that with addition of the correction term, the rescaled diffusion coefficient $D^{*}(\tau, T)$ follows super-Arrhenius behavior from a smaller value of $\tau$. The sensitivity to $\tau$ decreases significantly on correcting the expression, so $D^{*}(\tau, T)$ can provide a reasonable estimate of the true diffusion coefficient for shorter trajectories. However, the corrected diffusion constant is still too large for the smaller time windows, presumably because of the initial mean field assumption. As the negative correlation extends beyond adjacent steps for the small time windows (Fig. 3), we have also considered including higher order terms. However, although inclusion of higher order terms slightly improves the diffusion coefficient, the prediction is still far from the true value. Hence, we confirm that the mean field assumption is indeed not correct for very short time windows. We have estimated the mean diffusion coefficients for a range of densities for both the LJ and WCA systems (see the supplementary material). The overall results suggest that the incorporation of the negative correction is essential to recover super-Arrhenius behavior, independent of the density or the interatomic potential.
The attractive interaction is sometimes treated as a perturbation in liquid state theory. ${ }^{49}$ However, in the last decade, it has been established that the dynamical properties of models with purely repulsive interactions are less sluggish and heterogeneous compared to the full potential. ${ }^{33,50}$ The slowdown of the dynamical properties has been connected to the many-body correlations present in the system, ${ }^{51}$ although it has also recently been connected to the twobody structural properties. ${ }^{41,52}$ To investigate the role of fragility in the correction dynamics, we compare the diffusion coefficient $D(\tau, T)$ for the LJ and WCA systems at two distinct $\tau$ values and different densities. In Fig. 1, we can see that for the smaller value of $\tau$, the short time diffusion coefficient overestimates the actual diffusion constant, which is recovered for larger $\tau$ values. This result holds at higher densities for both systems, independent of the choice of time interval. In Fig. 6, we see that, at lower densities, even though the long-time diffusion coefficients are different for both systems, they exhibit similar behavior at smaller $\tau$. This result confirms that the models appear similar only if we ignore the negative correction term, suggesting that the difference in fragility is subsumed in the correction factor.

To quantify our observations, we plot the magnitude of the second term of Eq. (4) at $n=1$, since it is essentially zero otherwise. Interestingly, in Fig. 7, we observe that, as for the diffusion coefficient, the correction factor decreases with temperature and the rate of decrease qualitatively follows the same trend as the fragility. At higher densities, the magnitudes are close throughout the state points studied in the present work. This result is in good agreement with earlier studies, where at higher densities, the structures and dynamical properties were observed to behave similarly. ${ }^{41,52}$ The similar behavior of the temperature dependence of the correlation term and fragility has been quantified by plotting the long time diffusion coefficients and $\left\langle\cos \theta_{j, j+1}\right\rangle$ in Fig. 8. We observe a data collapse for the LJ and WCA systems at different densities, suggesting that the temperature dependences of the two quantities are strongly connected. Although our present study does not provide any analytical functional relationship, we see that the master plot holds for all the observation time windows. We have plotted $D(T)$ and $\left\langle\cos \theta_{j, j+1}\right\rangle$ at three different time windows for illustration. Our results show that for each system, density and observation time window, the temperature dependence of the diffusion coefficient always tracks the rate of change in the correlation function. (a) $\rho=1.2$

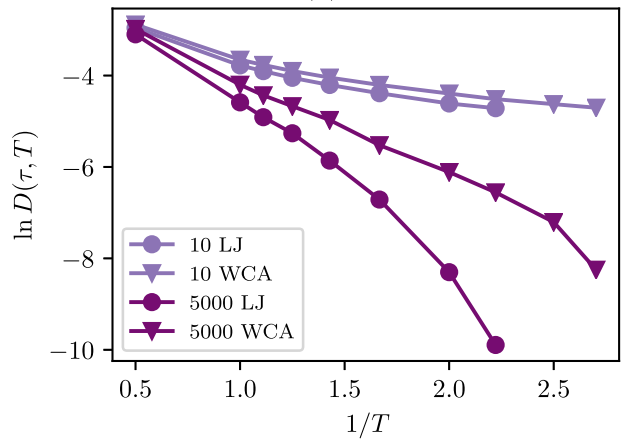

(b) $\rho=1.6$

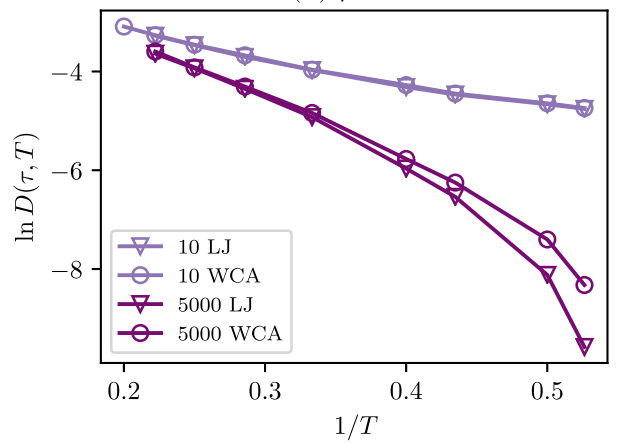

FIG. 6. The difference in fragility for $L J$ and WCA systems at $\rho=1.2$ and 1.6 is examined for two time intervals, $\tau=10$ and $\tau=5000$, by considering $\ln D(\tau, T)$. The principal difference in $D(\tau, T)$ arises at a low density for the larger $\tau$ value. 

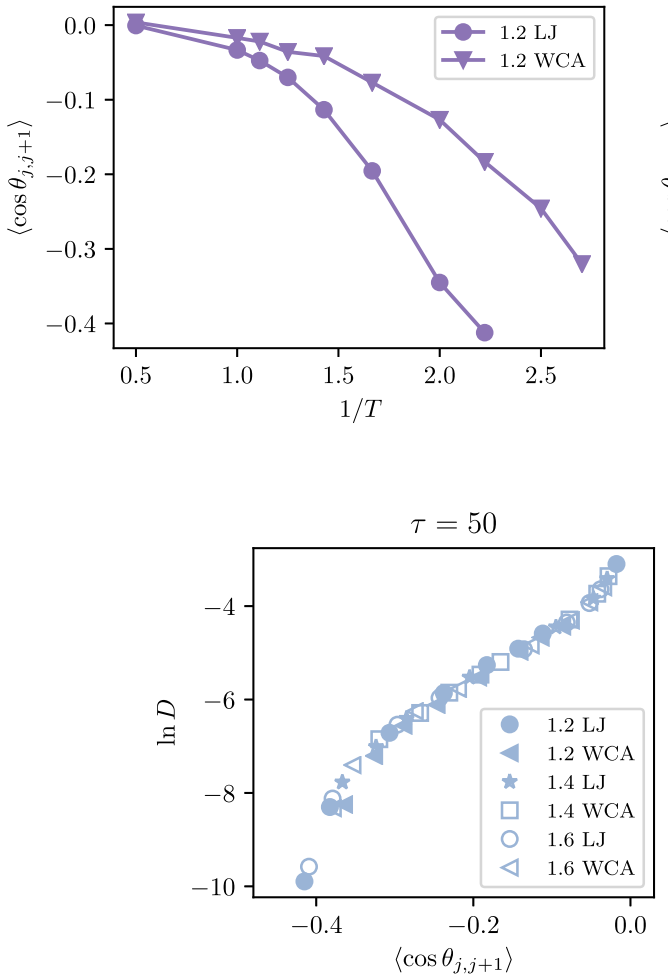

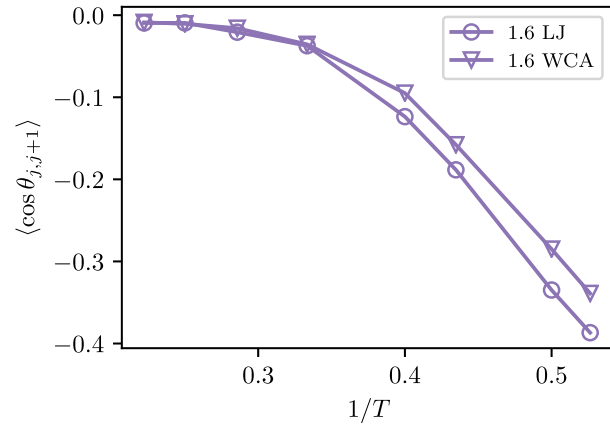

FIG. 7. The magnitude of $\left\langle\cos \theta_{j, j+1}\right\rangle$ increases on lowering the temperature for two selected densities. The temperature dependence of the anti-correlation factor follows a similar trend to the fragility.

FIG. 8. The true diffusion coefficient as a function of $\left\langle\cos \theta_{j, j+1}\right\rangle$ for three selected time windows. We observe a master plot for both systems at all the densities, which suggests a quantitative relationship between the diffusion coefficient and the correction function.

\section{CONCLUSION}

To investigate fragility and correlated dynamics, we have examined a binary Lennard-Jones system and its repulsive counterpart, WCA, over a range of densities. At lower temperatures, where both models follow super-Arrhenius behavior, we can estimate a nonergodic diffusion coefficient for short time windows, which exhibits Arrhenius-like behavior for both systems. For larger time windows, ergodicity is approached, and we recover the true super-Arrhenius behavior. The magnitude of the anti-correlation factor increases on lowering the temperature, suggesting that it is more important on supercooling. On combining the anti-correlation factor with the nonergodic diffusion coefficient, a mean field value is estimated, ${ }^{32}$ which is much closer to the true diffusion coefficient for both systems. This approach might be useful in future work to approximate the diffusion coefficients of ultrastable glasses in the deep supercooled region using a nonergodic trajectory. ${ }^{53-55}$ At lower densities, LJ and WCA have different fragilities. However, on short time scales, the nonergodic diffusion coefficients are similar, which supports the suggestion that the difference in fragility mainly originates from the correlation correction factor. The temperature dependence of the magnitude of the correction factor qualitatively behaves in a similar way to the fragility. At higher densities, LJ and WCA behave similarly in terms of both short and long time dynamics. The temperature dependence of the correction factor is also similar at higher densities. The correction factor reveals that the displacements are negatively correlated. The negative correction is directly manifested in the increased effective activation energy at lower temperatures for both models. The data collapse for both systems at different time windows strongly suggests a quantitative relationship between the rate of change of the negative correlation with temperature and the fragility.

\section{SUPPLEMENTARY MATERIAL}

See the supplementary material for the time-dependent diffusion coefficients $D(\tau, T)$ and the approximate diffusion coefficients $D^{*}(\tau, T)$ for LJ and WCA systems at $\rho=1.2,1.4$, and 1.6. We have also provided the behavior of the average correlation term for the two systems at all the densities studied in the present work.

\section{ACKNOWLEDGMENTS}

A.B. thanks Smarajit Karmakar and Sam Niblett for useful discussions. A.B. and D.J.W. thank the EPSRC for financial support.

\section{DATA AVAILABILITY}

The data that support the findings of this study are available from the corresponding author upon reasonable request. 


\section{REFERENCES}

${ }^{1}$ A. Cavagna, Phys. Rep. 476, 51 (2009).

${ }^{2}$ G. S. Fulcher, J. Am. Ceram. Soc. 8, 339 (1925).

${ }^{3}$ H. Vogel, Z. Phys. 22, 645 (1921).

${ }^{4}$ G. Tammann and W. Hesse, Z. Anorg. Allg. Chem. 156, 245 (1926).

${ }^{5}$ V. K. de Souza and D. J. Wales, Phys. Rev. B 74, 134202 (2006).

${ }^{6}$ J. C. Mauro, Y. Yue, A. J. Ellison, P. K. Gupta, and D. C. Allan, Proc. Natl. Acad. Sci. U. S. A. 106, 19780 (2009).

${ }^{7}$ L. Berthier and M. D. Ediger, J. Chem. Phys. 153, 044501 (2020).

${ }^{8}$ A. Angell, Nature 393, 521 (1998).

${ }^{9}$ K. Ito, C. T. Moynihan, and C. A. Angell, Nature 398, 492 (1999).

${ }^{10}$ C. A. Angell, J. Non-Cryst. Solids 131-133, 13 (1991).

${ }^{11}$ A. L. Greer, K. F. Kelton, and S. Sastry, Fragility of Glass-Forming Liquids (Hindustan Book Agency, New Delhi, 2014), Vol. TRIPS 13.

${ }^{12}$ S. P. Niblett, V. K. de Souza, J. D. Stevenson, and D. J. Wales, J. Chem. Phys. 145, 024505 (2016).

${ }^{13}$ S. P. Niblett, M. Biedermann, D. J. Wales, and V. K. de Souza, J. Chem. Phys. 147, 152726 (2017).

${ }^{14}$ E. R. Weeks, J. C. Crocker, A. C. Levitt, A. Schofield, and D. A. Weitz, Science 287, 627 (2000).

${ }^{15}$ K. Vollmayr-Lee, W. Kob, K. Binder, and A. Zippelius, J. Chem. Phys. 116, 5158 (2002).

${ }^{16}$ W. Kob, C. Donati, S. J. Plimpton, P. H. Poole, and S. C. Glotzer, Phys. Rev. Lett. 79, 2827 (1997).

${ }^{17}$ L. Berthier, G. Biroli, J.-P. Bouchaud, L. Cipelletti, D. El Masri, D. L'Hôte, F. Ladieu, and M. Pierno, Science 310, 1797 (2005).

${ }^{18}$ L. Berthier, G. Biroli, J.-P. Bouchaud, L. Cipelletti, and W. van Saarloos, Dynamical Heterogeneities in Glasses, Colloids, and Granular Media (OUP Oxford, 2011), Vol. 150.

${ }^{19}$ L. Berthier and G. Biroli, Rev. Mod. Phys. 83, 587 (2011).

${ }^{20}$ S. Karmakar, C. Dasgupta, and S. Sastry, Annu. Rev. Condens. Matter Phys. 5, 255 (2014).

${ }^{21}$ A. Rahman, Phys. Rev. 136, A405 (1964).

${ }^{22}$ G. Adam and J. H. Gibbs, J. Chem. Phys. 43, 139 (1965).

${ }^{23}$ E. Rabani, J. D. Gezelter, and B. J. Berne, Phys. Rev. Lett. 82, 3649 (1999).

${ }^{24}$ V. K. de Souza and D. J. Wales, J. Chem. Phys. 129, 164507 (2008).

${ }^{25}$ B. Doliwa and A. Heuer, Phys. Rev. E 67, 031506 (2003).

${ }^{26}$ A. Heuer, B. Doliwa, and A. Saksaengwijit, Phys. Rev. E 72, 021503 (2005).
${ }^{27}$ K. L. Ngai, J. Habasaki, D. Prevosto, S. Capaccioli, and M. Paluch, J. Chem. Phys. 137, 034511 (2012).

${ }^{28}$ S. Karmakar, C. Dasgupta, and S. Sastry, Phys. Rev. Lett. 116, 085701 (2016).

${ }^{29}$ I. Tah and S. Karmakar, Phys. Rev. Res. 2, 022067 (2020).

${ }^{30}$ K. Ngai, S. Capaccioli, M. Paluch, and L. Wang, "Clarifying the nature of the Johari-Goldstein $\beta$-relaxation and emphasising its fundamental importance," Philos. Mag. (published online, 2020).

${ }^{31}$ V. K. de Souza and D. J. Wales, J. Chem. Phys. 130, 194508 (2009).

${ }^{32}$ V. K. de Souza and D. J. Wales, Phys. Rev. Lett. 96, 057802 (2006).

${ }^{33}$ L. Berthier and G. Tarjus, Phys. Rev. Lett. 103, 170601 (2009).

${ }^{34}$ L. Berthier and G. Tarjus, J. Chem. Phys. 134, 214503 (2011).

${ }^{35}$ U. R. Pedersen, T. B. Schrèder, and J. C. Dyre, Phys. Rev. Lett. 105, 157801 (2010).

${ }^{36}$ A. Banerjee, S. Sengupta, S. Sastry, and S. M. Bhattacharyya, Phys. Rev. Lett. 113, 225701 (2014).

${ }^{37}$ F. Sciortino, W. Kob, and P. Tartaglia, J. Phys.: Condens. Matter 12, 6525 (2000).

${ }^{38}$ T. V. Bogdan, D. J. Wales, and F. Calvo, J. Chem. Phys. 124, 044102 (2006).

${ }^{39}$ D. J. Wales, Chem. Phys. Lett. 584, 1 (2013).

${ }^{40}$ F. P. Landes, G. Biroli, O. Dauchot, A. J. Liu, and D. R. Reichman, Phys. Rev. E 101, 010602 (2020).

${ }^{41}$ A. Banerjee, M. K. Nandi, S. Sastry, and S. M. Bhattacharyya, J. Chem. Phys. 145, 034502 (2016).

${ }^{42}$ S. Sengupta, T. B. Schrøder, and S. Sastry, Eur. Phys. J. E 36, 141 (2013).

${ }^{43}$ W. Kob and H. C. Andersen, Phys. Rev. E 51, 4626 (1995).

${ }^{44}$ J. D. Weeks, D. Chandler, and H. C. Andersen, J. Chem. Phys. 54, 5237 (1971).

${ }^{45}$ S. Plimpton, J. Comput. Phys. 117, 1 (1995).

${ }^{46}$ R. J. Hinde, R. S. Berry, and D. J. Wales, J. Chem. Phys. 96, 1376 (1992).

${ }^{47}$ D. J. Wales and R. S. Berry, J. Phys. B: At., Mol. Opt. Phys. 24, L351 (1991).

${ }^{48}$ T. Keyes and J. Chowdhary, Phys. Rev. E 64, 032201 (2001).

${ }^{49}$ J.-P. Hansen and I. R. McDonald, Theory of Simple Liquids (Elsevier, 1990).

${ }^{50}$ Z. Zhang, P. J. Yunker, P. Habdas, and A. Yodh, Phys. Rev. Lett. 107, 208303 (2011).

${ }^{51}$ G. Tarjus and C. Alba-Simionesco, arXiv:1401.2812 (2014).

${ }^{52}$ L. Wang, P. Guan, and W. H. Wang, J. Chem. Phys. 145, 034505 (2016).

${ }^{53}$ S. F. Swallen, K. L. Kearns, M. K. Mapes, Y. S. Kim, R. J. McMahon, M. D. Ediger, T. Wu, L. Yu, and S. Satija, Science 315, 353 (2007).

${ }^{54}$ A. D. Parmar, M. Ozawa, and L. Berthier, Phys. Rev. Lett. 125, 085505 (2020).

${ }^{55}$ P. Das, A. D. Parmar, and S. Sastry, arXiv:1805.12476 (2018). 